\title{
Mitigación y adaptación al cambio climático mediante la implementación de modelos integrados para el manejo y aprovechamiento de los residuos pecuarios. Revisión
}

\author{
Alberto Jorge Galindo-Barboza ${ }^{a^{*}}$ \\ Gerardo Domínguez-Araujo ${ }^{\text {a }}$ \\ Ramón Ignacio Arteaga-Garibay ${ }^{b}$ \\ Gerardo Salazar-Gutiérrez $^{\text {a }}$
}

\author{
a Instituto Nacional de Investigaciones Forestales, Agrícolas y Pecuarias (INIFAP). Campo \\ Experimental Centro Altos de Jalisco. México. \\ b INIFAP. Centro Nacional de Recursos Genéticos. Tepatitlán, Jalisco, México. \\ *Autor de correspondencia: galindo.alberto@inifap.gob.mx
}

\section{Resumen:}

La optimización del manejo y utilización de las excretas, se debe a que las especies animales no aprovechan el $100 \%$ de los nutrientes consumidos del alimento, siendo la excreta una fuente potencial de estos. La cantidad y calidad de la excreta depende de factores como el alimento, especie animal, etapa productiva, estado de salud y tipo de instalaciones. Los modelos integrados para manejo de residuos pecuarios, deben considerar la revalorización de estos como materia prima, con objeto de desarrollar tecnologías que habiliten la recuperación de nutrientes. El ensilaje de cerdaza, la composta, vermicomposta y los sistemas de digestión anaerobia forman parte de estos esquemas. Por otro lado, la importancia de la bio-remediación radica en la utilización del potencial metabólico de los microorganismos para transformar los contaminantes orgánicos, pudiéndose utilizar para limpiar espacios o aguas contaminadas. La estrategia de adopción tecnológica se diseña y se inicia a través de establecer las características del material a tratar, su acondicionamiento y las condiciones de operación del proceso, para seleccionar los criterios y métodos para su escalamiento en cualquier sistema de producción. 
Palabras clave: Materia prima, Excretas, Aprovechamiento, Ambiente, Sistemas Integrados, Bio-remediación.

Recibido: $14 / 11 / 2017$

Aceptado: 06/07/2018

\section{Introducción}

La población humana ha crecido el doble a partir de 1960, demandando alimentos y servicios; este crecimiento se refleja en los incrementos de los inventarios de población animal. En términos de alimentación, la producción de carne, leche y huevo, incrementan en proporción a los inventarios de producción animal; se estima que para el año 2020 se debe generar un aproximado de 200 billones de litros de leche y 100 millones de kilogramos de carne para satisfacer la demanda ${ }^{(1)}$. De la misma forma que la producción de alimentos, la generación de residuos orgánicos va en aumento, siendo el sector agropecuario un importante contribuyente a la contaminación atmosférica.

En 2013, a nivel global se estimó que del total de gases de efecto invernadero inducidos por el humano, el $14.5 \%$ (7.1 gigatoneladas de $\mathrm{CO}_{2}$-equivalente para 2005 y $10 \mathrm{Gt}$ para el año 2010) está representado por la cadena de suministro ganadero. De los cuales el $41 \%$ corresponde a la producción de carne de res, $20 \%$ a la leche, $9 \%$ a la producción de carne de cerdo, $8 \%$ a la producción de carne de pollo y huevo, $6 \%$ a la producción de leche y carne de pequeños rumiantes y el resto a otras especies de aves y rumiantes ${ }^{(2)}$.

En México, según el inventario nacional de emisiones de gases de efecto invernadero, en 2010 se estimó una emisión de 748.25 megatoneladas (Mt) de $\mathrm{CO}_{2}$-equivalente, de las cuales el $12.3 \%(92.18 \mathrm{Mt})$ corresponde a las emisiones por la agricultura, contribuyendo la fermentación entérica y el manejo de estiércoles con la emisión de $3.74 \mathrm{Mt}$ de $\mathrm{CO}_{2-}$ equivalente ${ }^{(2,3)}$.

De tal forma, los residuos pecuarios orgánicos representan una creciente y constante fuente de contaminantes. El poder contaminante de dichos residuos (estiércoles o excretas) radica en la presencia de nutrientes no digeridos, ya que ninguna especie aprovecha el total de los nutrientes consumidos en la dieta ${ }^{(4,5)}$. Por lo anterior se pueden considerar las excretas, una fuente potencial de nutrientes, los cuales pueden ser aprovechados mediante diversos procesos.

La producción y calidad de las excretas está ligada a factores como: especie, fin zootécnico, etapa productiva, calidad de las dietas, digestibilidad, entre otros. De igual manera, la 
infraestructura de la unidad de producción, el manejo y los equipos disponibles para su recolección, son factores que están ligados a las características físicas y químicas de los $\operatorname{residuos}^{(2,3)}$.

La caracterización de los residuos orgánicos pecuarios es pieza clave para planificar su manejo, aprovechamiento y disposición final y así, mitigar las emisiones y su efecto contaminante.

De tal forma, el objetivo de este trabajo es presentar los procesos que pueden ser adoptados bajo un modelo integrado para el aprovechamiento de residuos pecuarios preservando y reciclando los nutrientes derivados de los sistemas de producción animal.

\section{Modelos integrados para el manejo y aprovechamiento de residuos}

Los modelos integrados para el manejo y aprovechamiento de residuos consisten en la integración de tecnologías que nos lleven a tal fin. Es requisito de estos modelos su adaptabilidad a los distintos sistemas de producción pecuaria (familiar, mediana escala, gran escala, intensivos, extensivos y mixtos) y la interacción con la agricultura. Su principal objetivo es la diversificación de la producción y de los ingresos, estableciendo procesos amigables con el ambiente para alcanzar la sostenibilidad. El reto más importante y promisorio de estos modelos es la articulación con las cadenas productivas y comerciales locales y nacionales. En este sentido, son muchos, pero aislados, los esfuerzos que se han realizado para abordar distintas problemáticas, siendo la solución un enfoque integral de las necesidades en el sector agropecuario en relación al manejo de residuos.

Desde hace tiempo, se vienen realizando trabajos para identificar, cuantificar y tratar los residuos orgánicos de las explotaciones pecuarias, conceptualizándolos como un desecho, con la finalidad, de establecer estrategias y políticas para mitigar el impacto que tienen sobre el ambiente ${ }^{(6-12)}$.

Sin embargo, para la implementación de procesos sostenibles y la consolidación de esquemas integrados que mitiguen el impacto negativo sobre el ambiente y que además, generen estabilidad y rentabilidad, es de vital importancia revalorizar los residuos como materia prima $^{(2,13,14)}$ y como tal, es necesario determinar su disponibilidad, composición, características físicas y químicas $^{(15,16)}$ e inocuidad ${ }^{(17)}$; elementos mínimos inexcusables para determinar el nivel de aprovechamiento en los diversos procesos a los cuales puede ser sometida.

Actualmente se ha dado importancia a tecnologías que priorizan la recuperación de los nutrientes contenidos en los residuos pecuarios (Nitrógeno y Fosforo, principalmente), 
especialmente de los cerdos, con alternativas como la generación de biomasa proteica para uso en la alimentación animal ${ }^{(18)}$, sin embargo, a pesar de ser una alternativa viable para mitigar el impacto ambiental que generan los residuos, es limitada y no considera aspectos de inocuidad y toxicología.

Por otro lado, existen alternativas viables para lograr el objetivo de mitigar el impacto ambiental y aprovechar los residuos; sin embargo, la falta de capacitación para diseñarlos y operarlos, aunado a manejos inadecuados, encamina o sugiere su inutilidad. Un ejemplo de lo anterior son los sistemas de digestión anaerobia (biodigestores), los cuales, cuando no cumplen con el volumen adecuado, un régimen de carga establecido y retenciones hidráulicas adecuadas a las características de los residuos, generan efluentes que no pueden ser dispuestos para su aprovechamiento en la agricultura, debido a la alta concentración de nutrientes presentes ${ }^{(19)}$.

De tal manera, es indispensable generar, validar y adaptar tecnologías de acuerdo a las necesidades de una amplia gama de esquemas de producción agropecuaria, pero también a las características de la materia prima que se desee procesar y al objetivo productivo por alcanzar, considerando que, en este sentido, se encuentra implícito el ahorro en el costo ambiental de la producción. Para cumplir con lo anterior, es necesario la generación de modelos integrados, en los cuales, pueden estar incluidos uno o más procesos (tecnologías para el manejo de residuales) coadyuvando para un fin común.

En el Instituto Nacional de Investigaciones Forestales, Agrícolas y Pecuarias (INIFAP) se han diseñado y estudiado procesos para el acondicionamiento, manejo, aprovechamiento y revalorización de residuos orgánicos en el sector pecuario, entre los cuales se encuentran: ensilaje o fermentación de cerdaza para la alimentación animal ${ }^{(20-22)}$, composta y vermicomposta para la producción de abonos orgánicos ${ }^{(23)}$, sistemas de digestión anaerobia para la generación de energías renovables y el tratamiento de aguas residuales ${ }^{(24)}$. Estas tecnologías tienen la capacidad de: generar subproductos con valor agregado y acondicionar la materia prima para ser sometida a otro proceso del cual también se obtenga un subproducto o se prepare para un esquema de bio-remediación (Figura 1). 
Figura 1: Modelo de integración de procesos para el manejo y aprovechamiento de los residuos pecuarios

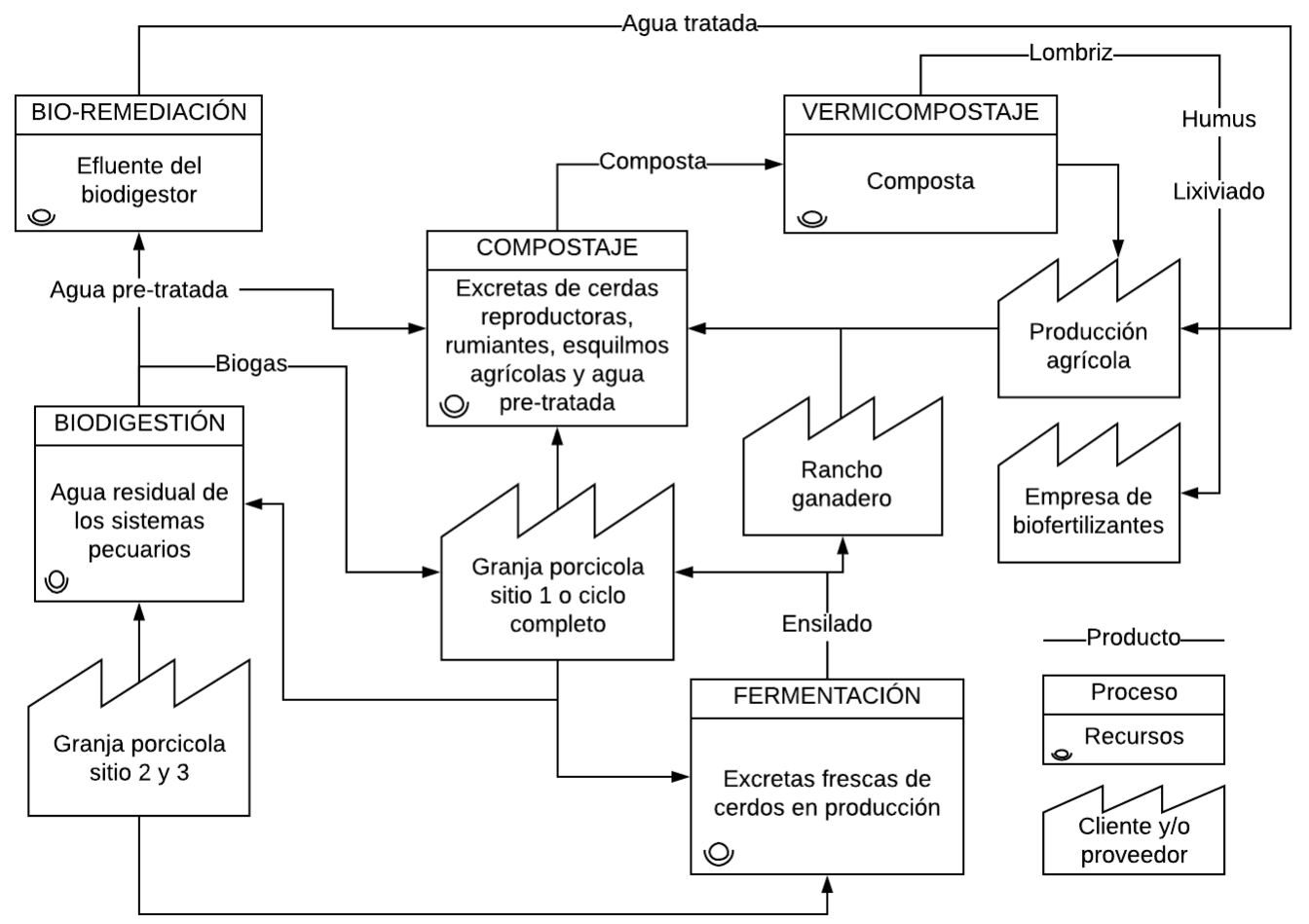

Los sistemas de producción agropecuarios son los proveedores de recursos (materia orgánica) para los procesos (alternativas tecnológicas) que generan productos comercializables, en donde un proveedor se puede convertir en cliente.

\section{Acondicionamiento de los residuos y generación de subproductos mediante procesos de ensilado o fermentación de cerdaza}

Desde hace tiempo, el uso de la cerdaza fresca o desecada, para la alimentación animal se ha venido dando, en cierta medida, debido a la falta de información sobre los riesgos y desventajas que representa, generando problemas de salud y probablemente exacerbando enfermedades; y aunque existen estudios en los cuales determinan que es factible su uso, solamente consideran aspectos productivos ${ }^{(25-29)}$ y no discurren aspectos concluyentes en relación a: salud animal, calidad de la canal, calidad y propiedades organolépticas de la leche, entre otros de importancia para el bienestar animal y la inocuidad; por otro lado, su inadecuado procesamiento genera un problema ambiental. 
Actualmente, el uso de cerdaza desecada, sigue teniendo auge con inclusiones de hasta un $70 \%$ en las dietas, con pérdidas de proteína cruda de hasta $12 \%$, respecto al contenido total de la excreta fresca ${ }^{(30,31)}$, lo que representa una limitante para el aprovechamiento de los nutrientes contenidos en la misma.

En función de las desventajas, riesgos y oportunidades que representa el uso de cerdaza para la alimentación animal, se ha desarrollado y perfeccionado un proceso para su acondicionamiento, denominado ensilaje de cerdaza o fermentación de cerdaza, el cual consiste en someter la excreta (proveniente de cerdos en etapas de destete-finalización) a un proceso de fermentación anaerobia ${ }^{(20)}$; el ensilado de cerdaza es el resultado final y puede ser utilizado para la alimentación de rumiantes ${ }^{(22,31-34)} \operatorname{cerdos}^{(35-38)}$ e incluso, dadas las características de este ingrediente, en otras especies como: peces, aves y conejos.

El principal objetivo de dicho proceso, es disminuir el $\mathrm{pH}$ a niveles por debajo de 5, con el fin de eliminar microorganismos indicadores de contaminación fecal ${ }^{(39)}$, proceso mediante el cual, también son eliminados microrganismos patógenos, virus y parásitos ${ }^{(17,40)}$, siempre y cuando, el proceso se realice adecuadamente. El mismo principio de fermentación anaerobia, es utilizado para el procesamiento de los residuos de alimentos humanos y su utilización en la alimentación de cerdos ${ }^{(41)}$ confiriendo ventajas sobre las propiedades químicas, físicas y microbiológicas para evitar su putrefacción.

En este sentido, el ensilado de cerdaza también se ha utilizado con el objetivo de conferir inmunidad en cerdos y disminuir el microbismo en granjas porcícolas ${ }^{(42)}$, sin que esto represente un riesgo, como el concebido al utilizar estrategias de auto inmunización como las realizadas en presencia de brotes por diarrea epidémica porcina ${ }^{(43)} \mathrm{u}$ otras enfermedades en México.

Es importante resaltar que el principal beneficio del ensilado de cerdaza, radica en la disminución de los costos de producción; trabajos recientes, sugieren una reducción en el costo de producción de hasta $7 \%$ con inclusiones del $30 \%$ en las etapas de crecimientodesarrollo-finalización de cerdos ${ }^{(21)}$ y de hasta $60 \%$ en la alimentación de rumiantes. Es trascendental considerar que el ensilado de cerdaza es un ingrediente de alta disponibilidad, para la formulación de dietas, por lo tanto, es indispensable conocer su composición química (la cual tiende a variar de acuerdo a la calidad de la materia prima utilizada) para formular en función de las necesidades nutricionales de los animales que se espere alimentar.

Cabe mencionar, que conforme se den investigaciones puntuales sobre los posibles usos y aplicaciones del ensilado de cerdaza, se obtendrá un aprovechamiento integral en beneficio de los distintos sistemas de producción pecuaria, sus productores y el ambiente. 


\section{Composta y vermi-composta}

A diferencia del ensilaje de cerdaza, en donde solamente se aprovechan las excretas de cerdos en etapas de destete-finalización, el compostaje, es un proceso versátil con el cual es posible acondicionar una gran cantidad de residuos agropecuarios. Si bien, el compostaje no es una práctica nueva, la adecuación de la técnica y el aceleramiento del proceso, lo hace innovador. Aunque en general, el compostaje es considerado un proceso sencillo, la práctica sugiere que necesita de condiciones físicas, químicas y microbiológicas complejas ${ }^{(23)}$ y la falta de cuidado o consideraciones repercuten sobre la calidad del producto final (composta estabilizada). La composta, posee un importante contenido de materia orgánica y nutrientes que pueden ser aprovechados de diversas formas en la agricultura y en la preservación del suelo ${ }^{(44,45)}$.

Para que el proceso de compostaje se lleve a cabo eficientemente y la composta sea rica en nutrientes, es importante considerar la calidad y la composición de las materias primas, en este sentido las excretas de cerdas en reproducción y rumiantes en general aportan características ideales para mezclarse con una extensa gama de esquilmos agrícolas de alta relación carbono-nitrógeno que son de difícil composteo, para su transformación y aprovechamiento $^{(13)}$.

Otra forma de utilizar y dar valor agregado a la composta, es a través de su aprovechamiento como insumo para la generación de vermi-composta mediante la lombricultura ${ }^{(46)}$. La lombricultura es considerada una biotecnología, en donde la lombriz funge como herramienta de trabajo para la transformación de residuos en productos orgánicos como la vermicomposta, la cual, contiene sustancias activas que actúan como reguladores de crecimiento en las plantas, tiene alto contenido de ácidos húmicos, y aumenta la capacidad de retención de humedad, lo que facilita la aireación y drenaje del suelo ${ }^{(23)}$, además, ostenta un alto contenido de potasio y fosforo ${ }^{(46,47)}$. También, la vermi-composta incrementa considerablemente la actividad microbial en el suelo y hay evidencia de que los reguladores en el crecimiento de las plantas como las citoquininas, auxinas, ácidos húmicos y microorganismos promueven el crecimiento de la planta independientemente de la suplementación de nutrientes ${ }^{(48,49)}$. Mediante la lombricultura, además de la vermi-composta, se obtienen otros sub-productos de alto valor económico, como lixiviados y biomasa de lombriz ${ }^{(44,46)}$.

Actualmente, se ha dado importancia a aspectos de eco-toxicología y seguridad del ambiente $^{(50)}$, analizando el riesgo que conlleva el uso de compostas en la generación de resistencia antimicrobiana ${ }^{(51)}$, degradación de antibióticos ${ }^{(52,53)}$, biodisponibilidad de metales pesados $^{(50)}$, emisión de gases ${ }^{(54)}$, persistencia de patógenos ${ }^{(55)}$, entre otros; sin embargo es importante considerar aspectos básicos, relacionados con la materia prima, a partir de la 
alimentación animal, manejo, sanidad y bioseguridad, los cuales son garantía de alimentos y residuos inocuos y de calidad.

\section{Sistemas de digestión anaerobia}

Los sistemas de digestión anaerobia son una alternativa viable para el pre-tratamiento de los residuos agropecuarios ${ }^{(56)}$. Su principal función consiste en degradar la materia orgánica y transformarla en metano; también se le ha dado uso a los efluentes como fertilizantes para terrenos de cultivo ${ }^{(57,58)}$. Lo anterior dependerá de la eficiencia del reactor (biodigestor).

Existen distintos tipos de biodigestores, entre los considerados de alta carga se encuentran: el reactor anaerobio discontinuo secuencial (ASBR, por sus siglas en inglés), y el reactor anaerobio de manto de lodos de flujo ascendente (UASB, por sus siglas en inglés), este tipo de biodigestores ofrece la ventaja de disminuir las cargas de sólidos de las aguas residuales en un tiempo relativamente corto, sin embargo, la inversión requerida es alta ${ }^{(59)}$.

En México, los biodigestores más utilizados para tratamiento de los efluentes de unidades de producción pecuaria son los de laguna cubierta ${ }^{(60)}$, de los cuales existen diversas versiones desarrolladas para facilitar su manejo y vida útil, mediante la implementación de sistemas de extracción de lodos y agitación ${ }^{(61)}$.

El manejo común y generalizado de este tipo de biodigestores, consiste en canalizar el $100 \%$ de los residuos sólidos generados en la unidad de producción mediante altos volúmenes de agua, en forma de "acarreo". Este tipo de prácticas se da, incluso, en zonas en donde existe una marcada escasez de agua, lo que representa un contrasentido para el fin de mitigar el impacto ambiental ${ }^{(62)}$. En consecuencia, se requieren biodigestores de gran tamaño que por ende requieren de gran espacio e inversión. Este tipo de biodigestores han demostrado, en algunos casos, eficiencias de un $78 \%$ hasta el $90 \%$ en la remoción de la demanda química de oxigeno ${ }^{(63)}$ y en la reducción total de huevos de helmintos ${ }^{(60)}$.

Otro tipo de biodigestores muy populares en México son los tubulares de polietileno (tipo Taiwán), los cuales, han sido eficientes en sistemas de traspatio para la generación y autoconsumo del biogás generado, sin embargo, este tipo de biodigestores bajo esquemas de producción pecuaria suelen quedar rebasados por la producción de residuos, sin que su adopción represente un beneficio. Además, en estudios recientes se ha demostrado que este tipo de biodigestores bajo flujo continuo en granjas porcícolas no son capaces de eliminar ciertos patógenos como: L. intracellularis, S. aureus, E. coli, Salmonella spp, mesófilos

aerobios, Clostridium sulfito reductores, coliformes totales y coccidias ${ }^{(64,65)}$, lo que hace que represente un riesgo sanitario el uso de sus efluentes como biol o fertilizante.

Por otro lado, diversos sectores (incluyendo el pecuario), han utilizado los biodigestores como generadores de energías renovables y algunas instituciones de investigación le han 
apostado al desarrollo e industrialización de esta tecnología. La utilización de diversas materias primas ${ }^{(66,67)}$, la conservación de materias primas para su utilización en la producción de biogás ${ }^{(68)}$, el desarrollo de sistemas de purificación, compresión y uso en motores de encendido por chispa ${ }^{(69)}$, son algunos de los tópicos de investigación.

Sin embargo, en el sector pecuario el principal fin de los biodigestores es la producción de energía eléctrica para auto-abastecer sus procesos productivos; en este sentido la producción porcícola es la más promisoria para tal fin, dadas las características de los residuos y sus particularidades en el sistema de producción. Esto da al sector una oportunidad competitiva en términos económicos, sociales y ambientales para la generación eléctrica ${ }^{(70,71)}$.

En los últimos años, la adopción de sistemas de biodigestión se ha vuelto popular entre los pequeños y medianos productores, los principales motivos corresponden a: la novedosa idea de generar biogás o energía, presión de las autoridades por establecer un proceso para el tratamiento de residuos, introducción al mercado de diseños económicos y a facilidades de financiamiento.

Sin embargo, antes de implementar un biodigestor (sin importar la escala de la unidad de producción), es necesario conocer la cantidad y características de los residuos generados para poder elaborar una estrategia de integración tecnológica y direccionar los residuos a cada uno de los procesos como mejor convenga. Si el biodigestor queda dentro de los procesos considerados, es de utilidad determinar su fin y su nivel de aprovechamiento, es decir, pretratamiento de aguas residuales, generación de energía (calorífica o eléctrica) o ambas. De esta manera se puede establecer su diseño-operación y se puede medir y maximizar su desempeño.

\section{Bio-remediación}

La bio-remediación es una rama de la bio-tecnología que utiliza el potencial metabólico de los microorganismos para transformar los contaminantes orgánicos en compuestos sin efectos o reducidos en sus efectos al mínimo y, por lo tanto, se puede utilizar para limpiar espacios o aguas contaminadas con perspectivas muy amplias ${ }^{(72,73)}$.

Sin embargo, existen algunas consideraciones relacionadas con la bio-remediación y que es importante mencionar; en comparación con los métodos químicos que se fundamentan en transferir la contaminación entre los tres estados físicos que presenta (estado gaseoso, líquido y sólido), en la bio-remediación se transfiere poca contaminación de un medio a otro debido a que es tecnología poco intrusiva y generalmente no requiere componentes estructurales o mecánicos dignos de destacar, además, es económicamente rentable, debido a que es un proceso natural con aceptación en contexto más allá de las implicaciones técnicas ${ }^{(74)}$. 
La bio-remediación presenta algunos inconvenientes y limitaciones. Por ejemplo, la biodegradación incompleta puede generar intermediarios metabólicos inaceptables, con un poder contaminante similar o incluso superior al producto de partida. Por otra parte, algunos compuestos, son resistentes o inhiben la bio-remediación. El tiempo requerido para el tratamiento adecuado puede ser difícil de predecir, además, el seguimiento, control de la velocidad y extensión del proceso es laborioso.

La eficiencia de esta técnica depende de varios factores como:

a) Propiedades del agente o agentes contaminantes (bio-degradabilidad).

b) Presencia de comunidades microbianas, con capacidad enzimática para metabolizar el o los compuestos. Los microorganismos pueden ser autóctonos (bio-remediación intrínseca o atenuación) $)^{(75)}$, añadidos al sistema para mejorar la degradación (bio-aumentación) o por suministro de condiciones óptimas que estimulan la actividad microbiana (bio-estimulación), suministro de oxígeno, nutrientes o modificaciones de $\mathrm{pH}$, entre otros.

c) Disponibilidad del contaminante. Es un factor crítico más importante que la propia presencia de comunidades microbianas. Para que la degradación de un contaminante pueda producirse, es necesario que interaccionen las células microbianas directamente con el contaminante, preferentemente en un medio acuoso ${ }^{(76)}$.

\section{Bio-remediación para descontaminación en residuos pecuarios}

La selección de procesos y el diseño de la estrategia de bio-remediación del agua y suelos contaminados con compuestos orgánicos como los residuos pecuarios, se inicia a través de establecer claramente las características del material a bio-remediar (efluentes de unidades de producción pecuaria o suelos contaminados), los microorganismos a utilizar, tipos de reactor (p. ej. digestores anaeróbicos o sistemas lagunares), pre-tratamiento del material contaminante (excretas principalmente, que se pueden pre-tratar o acondicionar con las alternativas antes citadas) y las condiciones de operación del proceso (dadas por el sistema de producción y el modelo integrado adoptado). Es necesario considerar también las evaluaciones por parte del laboratorio con objeto de explorar alternativas de operación, y cuantificar velocidades de degradación en función de parámetros críticos de operación como $\mathrm{pH}$, oxígeno y potencial de óxido-reducción, con la finalidad de determinar la eficiencia y eficacia del proceso de bio-remediación. A pequeña escala deben observarse los fenómenos fisicoquímicos, así como, determinar condiciones específicas para mejorar el proceso. Estos aspectos proporcionan una base importante para criterios y métodos de escalamiento de procesos (piloto, semi-comercial y comercial), así como los requerimientos de su instrumentación y control ${ }^{(77,78)}$. 


\section{Características de distribución de los contaminantes}

Antes de seleccionar cualquier alternativa de proceso de bio-remediación, debe caracterizarse muy bien el sitio o material a limpiar, hacer un estudio de pre-factibilidad técnica, económica y establecer claramente los aspectos físicos, químicos y microbiológicos. Para establecer con precisión los detalles de velocidad de limpieza, así como los factores que influyen en ello, para luego dar paso a la obtención de datos de cinética y equilibrio en mecanismos de reacción físicos, químicos y biológicos importantes para el diseño del proceso. Tener conocimiento del tipo de contaminante, su concentración, el grado de la problemática y la bio-disponibilidad sobre todo en procesos de lixiviación ${ }^{(79)}$.

\section{Determinación de los microorganismos a utilizar}

Las pruebas de degradación con distintos microorganismos son indispensables para determinar cual deberá ser utilizado, para esto se requiere información sobre el medio sobre el cual se dará el proceso (agua, suelo), contenido de materia orgánica, así como el perfil de distribución del tamaño de partícula, principalmente ${ }^{(77)}$. Los análisis microbiológicos incluyen parámetros como demanda bioquímica de oxígeno, determinación de cuenta viable, estudios de degradación in vitro previo al escalamiento del proceso ${ }^{(73)}$; y desde el punto de vista bioquímico, las rutas metabólicas involucradas durante la bio-degradación de los contaminantes y los posibles efectos benéficos o perjudiciales hacia el mismo proceso de degradación ${ }^{(80)}$. Es importante considerar las condiciones de temperatura, oxígeno, abastecimiento de nutrientes y la disponibilidad del contaminante, ya que pueden limitar las velocidades de degradación, principalmente al principio de los procesos en donde aún los factores limitantes no están bien definidos.

La experiencia dice que los mejores microorganismos para un proceso de bio-remediación están, precisamente, en el sitio a bio-remediar, es decir, deberá usarse, de preferencia, un microorganismo nativo ${ }^{(81)}$, sin embargo, es importante determinar la eficiencia y la velocidad de bio-degradación debido a que la concentración celular o de biomasa de microorganismos nativos, por lo general, es baja, o bien, porque no existen microorganismos capaces de biodegradar el material contaminante ${ }^{(76)}$, dando cabida a emplear un microorganismo de colección $^{(82,83)}$.

La bio-remediación de aguas residuales y suelos agropecuarios, con soporte microbiológico, bioquímico y de ingeniería, es una de las estrategias más promisorias para descontaminar estos recursos y es actualmente una alternativa más para los sistemas integrados de manejo y aprovechamiento de residuos y la incubación de negocios a través del uso apropiado de los resultados positivos que se generen de proyectos de investigación con aplicación tecnológica a costos reducidos y con beneficios tangibles hacia la población y el ambiente. 


\section{Conclusión}

La integración de tecnologías para el manejo y aprovechamiento de los residuos orgánicos pecuarios y la bio-rremediación de suelos y agua, es factible. Este tipo de modelos deberá articularse con los mercados locales, nacionales e internacionales y con las políticas ambientales para satisfacer la demanda de alimentos en cantidad y calidad, con la premisa de aprovechar y conservar los recursos naturales al máximo. Su adopción representa una oportunidad para obtener beneficios económicos, ambientales, sociales y tecnológicos.

\section{Literatura citada:}

1. Martinez J, Dabert P, Barrington S, Burton C. Livestock waste treatment systems for environmental quality, food safety, and sustainability. Bioresour Technol 2009;100(22):5527-36.

2. Gerber PJ, Steinfeld H, Henderson B, Mottet A, Opio C, Dijkman J, et al. Enfrentando el cambio climático a través de la ganadería. Una evaluación global de las emisiones y oportunidades de mitigación. Roma: Organización de las naciones unidas para la alimentación y la agricultura (FAO). 2013.

3. SEMARNAT. Secretaria de Medio Ambiente y Recursos Naturales. Inventario nacional de emisión de gases de efecto invernadero 1990-2010. Primera ed. México. 2013.

4. Brockmann D, Hanhoun M, Négri O, Hélias A. Environmental assessment of nutrient recycling from biological pig slurry treatment - Impact of fertilizer substitution and field emissions. Bioresour Technol 2014;(163):270-279.

5. Quiroga-Garza HM, Cueto-Wong JA, Figueroa-Viramontes U. Efecto del estiércol y fertilizante sobre la recuperación de $15 \mathrm{~N}$ y conductividad eléctrica. Terra Latinoam 2011;29(2):201-209.

6. Méndez-Novelo R, Castillo-Borges T, Vázquez-Borges E, Briceño-Pérez O, CoronadoPedaza V, Pat-Canuel R, et al. Estimación del potencial contaminante de las granjas porcinas y avícolas del estado de Yucatán. Ingeniería. 2009;13(2):13-21.

7. Pérez R. Porcicultura y contaminación del agua en La Piedad, Michoacán, México. Rev Int Contam Ambient 2001;17(1):5-13.

8. García A, León R, Míreles S, Castro JP, García AA, Roa JJ, et al. Contaminación ambiental en explotaciones porcinas mexicanas e incumplimiento de la norma ambiental. Rev Comput Prod Porc 2010;17(3):243-246. 
9. Jimenez Y, Negrin A, Valdés LA, Vidal V, Costa Y, Castro M, et al. Diagnostico de sistemas de tratamientos en el sector porcino no especializado de la provincia Ciego de Avila. Rev Comput Prod Porc. 2014;21(2):79-88.

10. Philippe FX, Nicks B. Review on greenhouse gas emissions from pig houses: Production of carbon dioxide, methane and nitrous oxide by animals and manure. Agric Ecosyst Environ 2015;199:10-25. doi.org/10.1016/j.agee.2014.08.015.

11. Bonilla-Cardenas JA, Lemus-Flores C. Emisión de metano entérico por rumiantes y su contribución al calentamiento global y al cambio climático. Revisión. Rev Mex Ciencias Pecu 2012;3(2):215-246.

12. Garzón-Zuñiga MA, Buelna G. Caracterización de aguas residuales porcinas y su tratamiento por diferentes procesos en México. Rev Int Contam Ambient 2014;30(1):65-79.

13. Dominguez-Araujo G, Galindo-Barboza AJ, Salazar-Gutiérrez G, Barrera-Camacho G, Sánchez-Garcia FJ. Las excretas porcinas como materia prima para procesos de reciclaje utilizados en actividades agropecuarias. Prometeo E. Tepatitlán de Morelos, Jalisco, México: Instituto Nacional de Investigaciones Forestales, Agrícolas y Pecuarias INIFAP; 2014.

14. Gómez-Rosales S, Angeles M de L, Espinosa-García JA, González-Orozco TA. Caracterización de sistemas de producción animal, manejo de excretas y opciones para su aprovechamiento integral. Querétaro, Querétaro: Instituto Nacional de Investigaciones Forestales, Agrícolas y Pecuarias INIFAP; 2008.

15. Anthony WB. Animal waste value-nutrient recovery and utilization. J Anim Sci 1971;32(4):799-802.

16. Bhattacharya AN, Taylor JC. Recycling animal waste as a feedstuff : A Review. J Anim Sci 1975;41(5):1438-1457.

17. Fontenot JP, Webb KEJ. Health aspects of recycling animal wastes by feeding. J Anim Sci 1975;40(6):1267-1276.

18. Mohedano RA, Velho VF, Costa RHR, Hofmann SM, Belli Filho P. Nutrient recovery from swine waste and protein biomass production using duckweed ponds (Landoltia punctata): Southern Brazil. Water Sci Technol 2012;65(11):2042-2048.

19. Jimenez-Peña Y, Valdés LA, Vidal-Olivera V, Castro-Carrillo M, Molineda A. Evaluación de efluentes anaerobios en el sector porcino no especializado de la provincia Ciego Ávila. Rev Comput Prod Porc 2014;21(3):140-145. 
20. Castellanos-Aceves A, Salazar-Gutiérrez G, Hernández-Morales P, Domínguez-Araujo G, Barrera-Camacho G. Uso de ensilado de cerdaza en la alimentacion animal. Prometeo Editores. Tepatitlán de Morelos, Jalisco, México: Instituto Nacional de Investigaciones Forestales, Agrícolas y Pecuarias INIFAP. 2010.

21. Galindo-Barboza AJ, Domínguez-Araujo G, Salazar-Gutiérrez G, Sánchez-Garcia FJ, Avalos-Castro MA. Uso de ensilado de cerdaza en la alimentacion animal. En: Hernández Virgen R, Pérez Domínguez JF, editores. Memoria tecnica Vamos al campo 2012. Prometeo E. Tepatitlán de Morelos, Jalisco, México: Instituto Nacional de Investigaciones Forestales, Agrícolas y Pecuarias INIFAP; 2012:57-62.

22. Avalos-Castro MA, Dominguez-Araujo G, Galindo-Barboza AJ, Ruvalcaba-Gomez JM, Arias-Chávez LE, Salazar-Gutiérrez G. Efecto de la adición de ensilado de cerdaza en la dieta de vacas en lactación sobre paramétros productivos y las caracteristicas fisicoquímicas de la leche. Congreso Nacional, Mitigación del daño ambiental en el sector agropecuario de México. 2013:127-136.

23. Xelhuantzi-Carmona J, Salazar-Gutiérrez G, Dominguez-Araujo G, Arias-Chávez LE, Chávez-Durán ÁA, Galindo-Barboza AJ. Manual para la elaboración de abonos organicos apartir de técnicas como la composta y lombricomposta. Graficos Lara editores. Tepatitlán de Morelos, Jalisco, México: Instituto Nacional de Investigaciones Forestales, Agrícolas y Pecuarias INIFAP; 2012. 52 p.

24. Dominguez-Araujo G, Salazar-Gutiérrez G, Galindo-Barboza AJ, Xelhuantzi-Carmona J, Castañeda-Castillo M, Sánchez-Garcia FJ, et al. Implementación de biodigestores para pequeños y medianos productores porcícolas. Graficos Lara editores. Tepatitlán de Morelos, Jalisco, México: Instituto Nacional de Investigaciones Forestales, Agrícolas y Pecuarias INIFAP; 2012. 28 p.

25. Padilla-Goyo EC, Castellanos-Ruelas AF, Cantón-Castillo JG, Moguel-Ordoñez YB. Impacto del uso de niveles elevados de excretas animales en la alimentación de ovinos. Livest Res Rural Dev 2000;12(1):14-22.

26. Ortega-Zuñiga I. Digestibilidad in vivo en ovinos de dietas con dos niveles de cerdaza y melaza. [tesis licenciatura]. Zapopan, Jalisco. Universidad de Guadalajara; 1996.

27. Newton GL, Utley PR, Ritter RJ, McCorminck WC. Performance of beef cattle fed wastelage and digestibility of wastelage and dried waste diets. J Anim Sci 1977;(44):447-451.

28. Mejía W, Quintero A, Rodríguez E, Calatayud D. Efecto de la administración de cerdaza sobre el rendimiento productivo de cerdos en etapa de engorde. Arch Latinoam Prod Anim 1997;5(1):300-301. 
29. Heredia-Cruz MR. Utilización de cerdaza en dietas de levante para terneros pos destete [tesis licenciatura]. Zamorano, Honduras: Escuela Agrícola Panamericana; 2012.

30. Orellana-González CA, Dimas-Fontanals JA. Evaluación y formulación de un concentrado a partir de cedaza como fuente de proteina para engorde de conejos y pollos broiler [tesis licenciatura]. Antiguo Cuscatlán, El Salvador. Universidad Dr. Jose Matias Delgado; 2009.

31. Castrillón-Quintana O, Jiménez-Pérez RA, Bedoya-Mejía O. Porquinaza en la alimentación animal. Rev Lasallista Investig 2004;1(1):1-5.

32. Berger JCA, Fontenot JP, Kornegay ET, Webb KEJ. Feeding swine waste. II. Nitrogen utilization, digestibility and palatability of ensiled swine waste and Orchardgrass hay or corn grain fed to sheep. J Anim Sci 1981;(52):1404-1420.

33. Dominguez-Araujo G, Galindo-Barboza AJ, Salazar-Gutiérrez G, Arias-Chávez LE, Quiñones-Islas N. Efecto en el comportamiento productivo de bovinos en finalización al utilizar dietas conteniendo ensilado de cerdaza. Rev Mitigación del Daño Ambient Agroaliment y For México. 2014;1(1):23-33.

34. Martínez-Barrera VM, Serna-Roman MG. Utilización de cerdaza ensilada en la alimentación de ovinos de engorda [tesis licenciatura]. Zapopan, Jalisco, México. Universidad de Guadalajara; 1999.

35. Guillemin Rubio JJ. Utilizacion de la cerdaza fermentada en la etapa de destete [tesis licenciatura]. Zapopan, Jalisco, México. Universidad de Guadalajara; 1995.

36. Galindo-Barboza AJ, Dominguez-Araujo G, Salazar-Gutiérrez G, Avalos-Castro MA, Sánchez-Garcia FJ. Efecto de la adición de ensilado de cerdaza en dietas de cerdos en cebo, una alternativa para la reutilización de solidos en granjas porcícolas. Congreso Nacional, Mitigación del daño ambiental en el sector agropecuario de México. Guadalajara, Jalisco, México; 2013:61-71.

37. Zaldivar-Reynoso MA, Corona-Santos G. Restricción del consumo de alimento en cerdos en atapa de finalización, utilizando estiercol fermentado de cerdo [tesis licenciatura]. Zapopan, Jalisco, México. Universidad de Guadalajara; 1995.

38. Hernandez-Mata A. Evaluacion de solidos recuperados fermentados con alimento de lechon de predestete y efectos en su aceptacion y crecimiento [tesis licenciatura]. Guadalajara, Jalisco, México.Universidad de Guadalajara; 1994.

39. Galindo-Barboza AJ, Dominguez-Araujo G, Salazar-Gutiérrez G, Arteaga-Garibay RI, Martínez-Peña MD, Ruvalcaba-Gomez JM. Disminución de las UFC como indicadores de contaminación fecal en el ensilado de cerdaza, considerando el $\mathrm{pH}$ como factor 
determinante. Rev Mitigación del Daño Ambient Agroaliment y For México 2014;1(1):34-43.

40. Caballero-Hernández AI, Castrejón-Pineda F, Martínez-Gamba R, Angeles-Campos S, Pérez-Rojas M, Buntinx SE. Survival and viability of Ascaris suum and Oesophagostomum dentatum in ensiled swine faeces. Bioresour Technol 2004;94(2):137-142.

41. Yang SY, Ji KS, Baik YH, Kwak WS, McCaskey TA. Lactic acid fermentation of food waste for swine feed. Bioresour Technol 2006;97(15):1858-1864.

42. Galindo-Barboza AJ, Dominguez-Araujo G, Salazar-Gutiérrez G, Arteaga-Garibay RI, Martinez-Peña MD, Sanchez-Garcia FJ. Ensilado de cerdaza, una oportunidad para el manejo de la bioseguridad y el microbismo en granjas porcícolas. Prometeo Editores. Tepatitlán de Morelos, Jalisco, México: Instituto Nacional de Investigaciones Forestales, Agrícolas y Pecuarias INIFAP; 2013.

43. Jung K, Saif LJ. Porcine epidemic diarrhea virus infection: Etiology, epidemiology, pathogenesis and immunoprophylaxis. Vet J 2015;204(2):134-143.

44. FAO. Manual de compostaje del agricultor, Experiencias en América Latina. Oficina Regional de la FAO para América Latina y el Caribe. 2013. http://www.fao.org/3/ai3388s.pdf

45. Villar I, Alves D, Garrido J, Mato S. Evolution of microbial dynamics during the maturation phase of the composting of different types of waste. Waste Manag 2016;(54):83-92.

46. Gómez-Rosales S, Espinosa-García JA, González-Orozco TA, Salazar-Gutiérrez G. Alternativas para el reciclaje de excretas animales: Producción de humus de lombriz. Impresos G. editores. Ajuchitlán, Colon, Queretaro: Instituto Nacional de Investigaciones Forestales, Agrícolas y Pecuarias INIFAP; 2007.

47. Delgado-Arrollo MM, Porcel-Cots MÁ, Miralles de Imperal-Hornedo R, BeltránRodríguez EM, Beringola-Beringola L, Martín-Sánchez JV. Efecto de la vermicultura en la descomposición de residuos orgánicos. Rev Int Contam Ambient 2004;20(2):8386.

48. Chaoui HI, Zibilske LM, Ohno T. Effects of earthworm casts and compost on soil microbial activity and plant nutrient availability. Soil Biol Biochem 2003;35(2):295302.

49. Masciandaro G, Ceccanti B, Garcia C. "In situ” vermicomposting of biological sludges and impacts on soil quality. Soil Biol Biochem 2000;32(7):1015-1024. 
50. Wang Q, Wang Z, Awasthi MK, Jiang Y, Li R, Ren X, et al. Evaluation of medical stone amendment for the reduction of nitrogen loss and bioavailability of heavy metals during pig manure composting. Bioresour Technol 2016;(220):297-304.

51. Kang Y, Hao Y, Shen M, Zhao Q, Li Q, Hu J. Impacts of supplementing chemical fertilizers with organic fertilizers manufactured using pig manure as a substrate on the spread of tetracycline resistance genes in soil. Ecotoxicol Environ Saf 2016;(130):279288.

52. Selvam A, Wong JWC. Degradation of antibiotics in livestock manure during composting. In: Wong JW-C, Tyagi RD, Pandey A. Current developments in biotechnology and bioengineering. Elsevier; 2017:267-292.

53. Xie W-Y, Yang X-P, Li Q, Wu L-H, Shen Q-R, Zhao F-J. Changes in antibiotic concentrations and antibiotic resistome during commercial composting of animal manures. Environ Pollut 2016;(219):182-190.

54. Zang B, Li S, Jr. FM, Li G, Luo Y, Zhang D, et al. Effects of mix ratio, moisture content and aeration rate on sulfur odor emissions during pig manure composting. Waste Manag 2016;(56):498-505.

55. Hénault-Ethier L, Martin VJJ, Gélinas Y. Persistence of Escherichia coli in batch and continuous vermicomposting systems. Waste Manag 2016;(56):88-99.

56. Trejo LW, Vázquez GLB, Uicab AJ, Castillo CJ, Caamal MA, Belmar CR, Santos RRH. Eficacia de remoción de materia orgánica de aguas residuales porcinas con biodigestores en el estado de Yucatán, México. Trop Subtrop Agroecosystems 2014; (17): 321-323.

57. Acevedo P. Biodigestor de Doble Proposito - Producción e Investigación - para residuos de granja porcicola. Revista Ion 2006;19(1):1-6.

58. Cepero L, Savran V, Blanco D, Díaz PMR, Suarez J, Palacios A. Producción de biogás y bioabonos a partir de efluentes de biodigestores. Pastos y Forrajes 2012;35(2):219_ 226.

59. Pérez-Pérez T, Pereda-Reyes I, Oliva-Merencio D, Zaiat M. Anaerobic digestion technologies for the treatment of pig wastes. Cuba J Agric Sci 2016;50(3):343-354.

60. Blanco D, Suárez J, Jiménez J, González F, Álvarez MN, Cabeza E, et al. Eficiencia del tratamiento de residuales porcinos en digestores de laguna tapada. Pastos y Forrajes 2015;38(4):441-447.

61. Campos CB. Metodología para determinar los parámetros de diseño y construcción de biodigestores para el sector cooperativo y campesino. Rev Cienc Téc Agropec 2011;20(2):37-41. 
62. Pérez PT, Pereda RI, Oliva MD, Zaiat M. Anaerobic digestion technologies for the treatment of pig wastes. Cuban J Agr Sci 2016;50(3):343-354.

63. Orrico J, Marco AP, Orrico A, De Lucas JJ. Influência da relação volumoso: concentrado e do tempo de retenção hidráulica sob a biodigestão anaeróbia de dejetos de bovinos. Engenharia Agrícola 2010;(30):386-394.

64. Betancur HO, Betancourt EA, Estrada AJ, Henao UF. Persistence of pathogens in liquid pig manure processed in manure tanks and biodigesters. Rev MVZ Córdoba 2016;21(1):5237-49.

65. Cañon-Franco WA, Henao-Agudelo RA, Pérez-Bedoya JL. Recovery of gastrointestinal swine parasites in anaerobic biodigester systems. Rev Bras Parasitol Veterinária 2012;21(3):249-53.

66. Angelidaki I, Alves M, Bolzonella D, Borzacconi L, Campos JL, Guwy AJ, et al. Defining the biomethane potential (BMP) of solid organic wastes and energy crops: a proposed protocol for batch assays. Water Sci Technol 2009;59(5):927-934.

67. Mustafa SS, Mustafa SS, Mutlag AH. Kirkuk municipal waste to electrical energy. Int J Electr Power Energy Syst. 2013;44(1):506-513.

http://dx.doi.org/10.1016/j.ijepes.2012.07.053

68. Teixeira Franco R, Buffière P, Bayard R. Optimizing storage of a catch crop before biogas production: Impact of ensiling and wilting under unsuitable weather conditions. Biomass and Bioenergy 2017;(100):84-91.

69. Porpatham E, Ramesh A, Nagalingam B. Investigation on the effect of concentration of methane in biogas when used as a fuel for a spark ignition engine. Fuel 2008;87(89):1651-1659.

70. Venegas Venegas JA, Espejel García A, Pérez Fernández A, Castellanos Suárez JA, Sedano Castro G. Potencial de energía eléctrica y factibilidad financiera para biodigestor-motogenerador en granjas porcinas de Puebla. Rev Mex Cienc Agr 2017;8(3):735-740.

71. Venegas Venegas JA, Perales Salvador A, del Valle Sánchez M. Energía renovable una opción de competitividad en granjas porcinas en México. Rev Mex Cienc Agr 2015;(1):503-509.

72. Glazer AN, Nikaido H. Microbial biotechnology: Fundamentals of applied microbiology. New York, USA: WH Freeman and Company; 1995.

73. Atlas RM, Bartha R. Ecología microbiana \& microbiología ambiental. Pearson Educación, Madrid. 2001. 
74. Frutos FJG, Escolano O, García S, Mar Babín M, Fernández MD. Bioventing remediation and ecotoxicity evaluation of phenanthrene-contaminated soil. J Hazard Mater 2010;(183):806-813.

75. Rosenberg E, Ron EZ. Bioremediation of petroleum contamination. In: Crawford RL \&Crawford DL editors. Bioremediation. Principles and applications biotechnology research series 6. University Press, Cambridge. 1996:100-124.

76. Azubuike CC, Chikere CB, Okpokwasili GC. Bioremediation techniques-classification based on site of application: principles, advantages, limitations and prospects. World J Microbiol Biotechnol 2016;32(11):180.

77. Verma JP, Jaiswal DK. Book review: advances in biodegradation and bioremediation of industrial waste. Front Microbiol 2016;(6):1-2.

78. Firmino PIM, Farias RS, Barros AN, Buarque PMC, Rodriguez E, Lopes AC, et al. Understanding the anaerobic BTEX removal in continuous-flow bioreactors for ex situ bioremediation purposes. Chem Eng J 2015;(281):272-280.

79. Smith E, Thavamani P, Ramadass K, Naidu R, Srivastava P, Megharaj M. Remediation trials for hydrocarbon-contaminated soils in arid environments: evaluation of bioslurry and biopiling techniques. Int Biodeterior Biodegradation 2015;(101):56-65.

80. Khan FI, Husain T, Hejazi R. An overview and analysis of site remediation technologies. J Environ Manag 2004;(71):95-122.

81. Philp JC, Atlas RM. Bioremediation of contaminated soils and aquifers. In: Atlas RM, Philp JC, editors Bioremediation: applied microbial solutions for real-world environmental cleanup. American Society for Microbiology (ASM) Press, Washington, 2005:139-236.

82. Mohan SV, Sirisha K, Rao RS, Sarma PN. Bioslurry phase remediation of chlorpyrifos contaminated soil: process evaluation and optimization by Taguchi design of experimental (DOE) methodology. Ecotoxicol Environ Saf 2007;(68):252-262.

83. Zangi-Kotler M, Ben-Dov E, Tiehm A, Kushmaro A. Microbial community structure and dynamics in a membrane bioreactor supplemented with the flame retardant dibromoneopentyl glycol. Environ Sci Pollut Res Int 2015;(22):17615-17624. 